\title{
Epitaxial Lead Chalcogenides on Si for Mid-IR Detectors and Emitters Including Cavities
}

\section{Journal Article}

\section{Author(s):}

Zogg, Hans; Arnold, Martin; Felder, Ferdinand; Rahim, Mohamed; Ebneter, Christian; Zasavitskiy, Ivan; Quack, Niels; Blunier, Stefan; Dual, Jürg

\section{Publication date:}

2008

\section{Permanent link:}

https://doi.org/10.3929/ethz-b-000011685

\section{Rights / license:}

In Copyright - Non-Commercial Use Permitted

\section{Originally published in:}

Journal of Electronic Materials 37(9), https://doi.org/10.1007/s11664-008-0429-0 


\title{
Epitaxial Lead Chalcogenides on Si for Mid-IR Detectors and Emitters Including Cavities
}

\author{
H. ZOGG,${ }^{1,4}$ M. ARNOLD,${ }^{1}$ F. FELDER,${ }^{1}$ M. RAHIM ${ }^{1}$ C. EBNETER, ${ }^{1}$ \\ I. ZASAVITSKIY, ${ }^{2}$ N. QUACK, ${ }^{3}$ S. BLUNIER, ${ }^{3}$ and J. DUAL ${ }^{3}$ \\ 1.-Thin Film Physics Group, ETH Zurich, Technopark, 8005 Zurich, Switzerland. 2.-P.N. \\ Lebedev Physical Institute, Russian Academy of Sciences, Moscow 117924, Russia. 3.-Institute of \\ Mechanical Systems, ETH Zurich, Zurich 8092, Switzerland. 4.-e-mail: zogg@phys.ethz.ch
}

Lead chalcogenide (IV-VI narrow-gap semiconductor) layers on $\mathrm{Si}$ or $\mathrm{BaF}_{2}(111)$ substrates are employed to realize two mid-infrared optoelectronic devices for the first time. A tunable resonant cavity enhanced detector is realized by employing a movable mirror. Tuning is across the $4 \mu \mathrm{m}$ to $5.5 \mu \mathrm{m}$ wavelength range, and linewidth is $<0.1 \mu \mathrm{m}$. Due to the thin $(0.3 \mu \mathrm{m}) \mathrm{PbTe}$ photodiode inside the cavity, a higher sensitivity at higher operating temperatures was achieved as compared to conventional thick photodiodes. The second device is an optically pumped vertical external-cavity surface-emitting laser with $\mathrm{PbTe}$-based gain layers. It emits at $\sim 5 \mu \mathrm{m}$ wavelength and with output power up to $50 \mathrm{~mW}$ pulsed, or $3 \mathrm{~mW}$ continuous wave at $100 \mathrm{~K}$.

Key words: Mid-infrared optoelectronic devices, VECSEL, RCED, lead chalcogenides, epitaxy

\section{INTRODUCTION}

Narrow-gap lead chalcogenides (IV-VI compounds) such as $\mathrm{Pb}_{1-x} \mathrm{Sn}_{x} \mathrm{Te}$ (LTT) or $\mathrm{Pb}_{1-x} \mathrm{Sn}_{x} \mathrm{Se}$ (LTS) have been known for a long time and were employed as early as the 1970s to fabricate infrared detector arrays for thermal imaging. ${ }^{1}$ They can cover the infrared (IR) range from $<3 \mu \mathrm{m}$ to $>30 \mu \mathrm{m}$ by choosing appropriate chemical compositions. The properties of IV-VI materials are remarkably different from the well-known II-VI or III-V compounds (Table I): in narrow-gap IV-VI compounds, the minimal (direct) bandgap occurs at the L-point of the Brillouin zone, and the valence and conduction bands are nearly mirror images of each other, leading to nearly equal electron and hole masses. The ultimate sensitivities obtainable with IV-VI compounds are similar to those of II-VI narrowbandgap semiconductors such as $\mathrm{Cd}_{1-x} \mathrm{Hg}_{x} \mathrm{Te}$ under similar conditions (i.e., bandgap and operating temperature). They are limited by Auger recombination in both cases. This leads to maximal $R_{\mathrm{o}} A$

(Received November 8, 2007; accepted February 26, 2008; published online August 6, 2008) (differential resistance at zero bias times area) products over a certain doping concentration: e.g., optimal concentrations for $0.1 \mathrm{eV}$ bandgap devices at $80 \mathrm{~K}$ are $\sim 2 \times 10^{14} \mathrm{~cm}^{-3}$ to $5 \times 10^{15} \mathrm{~cm}^{-3}$ for $\mathrm{Hg}_{1-x}$ $\mathrm{Cd}_{x}$ Te (MCT) and $\sim 1 \times 10^{17} \mathrm{~cm}^{-3}$ to $3 \times 10^{17} \mathrm{~cm}^{-3}$ for LTS or LTT. The much higher optimal concentrations in LTT and LTS are due to the larger homogeneity region and high permittivities in these materials. These high permittivities, as well as the high thermal expansion coefficients (a factor of 7 higher than $\mathrm{Si}$ ), were one reason why development for IR focal-plane array (FPA) thermal imaging was nearly entirely stopped in the early 1980s in favour of MCT. However, IV-VI materials remained the only choice to fabricate mid-wave infrared (MWIR) laser diodes before the invention of the quantum cascade laser, ${ }^{2}$ and continue to be of importance today.

Renewed interest started in the mid-1980s with the growth of IV-VI epitaxial layers on non-latticematched substrates such as Si. High-quality layers were obtained by molecular-beam epitaxy (MBE) on $\mathrm{Si}(111)$ substrates employing a very thin $\mathrm{CaF}_{2}$ buffer layer for compatibility. Of most importance is 
Table I. Some Properties of IV-VI Materials Versus MCT

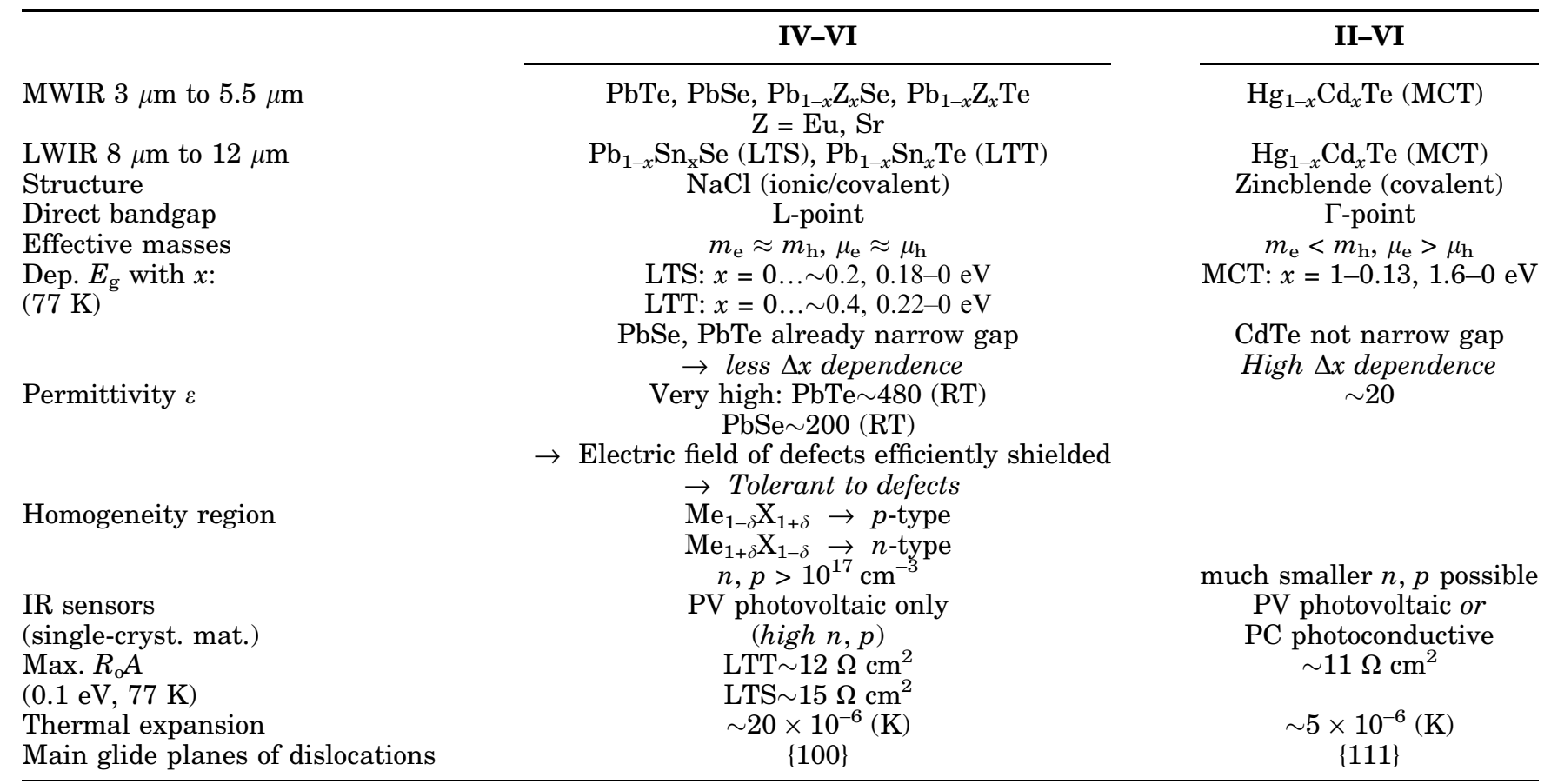

the relief of the thermal mismatch strain by glide of dislocations: the dislocations glide on $\{100\}$ planes, which are inclined with respect to the (111) surface plane, i.e., the Schmid factors and therefore the shear strains are not zero. On each temperature change, the threading ends of the misfit dislocations glide to remove the mechanical thermal strain built up. They never block (as might occur in zincblendetype II-VI and III-V semiconductors) and in addition may even react to decrease the density of threading dislocations and therefore increase the structural layer quality. ${ }^{3}$ Linear IV-VI IR sensor arrays on $\mathrm{Si}(111)$ were demonstrated with cut-off wavelength ranging from $\sim 3 \mu \mathrm{m}$ to $\sim 15 \mu \mathrm{m} .{ }^{4}$ A twodimensional (2D) FPA with the IV-VI layer grown epitaxially on an active Si chip containing the readout multiplexing electronics was also demonstrated, proving the concept that whole IR FPAs might be fabricated by postprocessing of complete and tested Si-complementary metal oxide semiconductor (Si-CMOS) circuits. ${ }^{5}$

An additional application for IV-VI optoelectronic devices emerged in the late 1990s. Choosing IV-VI compositions $\mathrm{Pb}_{1-x} \mathrm{Y}_{x} \mathrm{Z}$ with $\mathrm{Y}=\mathrm{Sr}$ or $\mathrm{Eu}(\mathrm{Z}=\mathrm{Te}$ or Se), layers with higher bandgap are obtained. Even a few percent of $\mathrm{Eu}$ or $\mathrm{Sr}$ suffices to increase the bandgap significantly, while with still higher compositions $(x \rightarrow 1)$ bandgaps of up to $2 \mathrm{eV}$ result. Parallel to the increased bandgap values, the refractive indices decrease significantly. This allows a high freedom to design more-elaborate device structures including epitaxial Bragg mirrors, which can easily be realized by MBE with IV-VI materials.
Bragg mirrors consist of quarter-wavelength pairs with alternating high- and low-refractive-index layers. Their refractive index $n$ is very large $(n \approx 5$ to 6 ) for the narrow-band compositions, while, e.g., for EuSe $n=2.4$, or $\mathrm{BaF}_{2}$ (which is also suitable) $n=1.4$. Due to this high index contrast, a few quarter-wavelength pairs suffice to obtain near $100 \%$ reflectivity across a broad spectral band. ${ }^{6}$

In the following, we describe two applications that combine both IR active layers and Bragg mirrors: tunable resonant cavity enhanced detectors (RCEDs) and vertical external-cavity surfaceemitting lasers (VECSEL).

\section{TUNABLE RESONANT CAVITY ENHANCED DETECTORS}

The RCED principle allows IR detectors with sensitivity only in a narrow spectral band to be obtained. ${ }^{7}$ Single elements may be applied for mid-IR microspectrometers, while with whole $2 \mathrm{D}$ arrays adaptive focal-plane arrays (AFPA) may be realized. For both applications, a very thin active layer is grown inside a Fabry-Perot cavity formed by two mirrors. RCEDs offer much higher peak quantum efficiency as compared to broadband detectors with an external line filter. ${ }^{8}$ In addition, the Johnson noise level is lower due to the smaller active detector volume.

We already reported on realizations of such RCED for the mid-IR range at $\sim 4 \mu \mathrm{m}$ to $5 \mu \mathrm{m}$ peak wavelengths. ${ }^{9}$ They operate at lower noise levels at suitable operating temperatures as compared to 


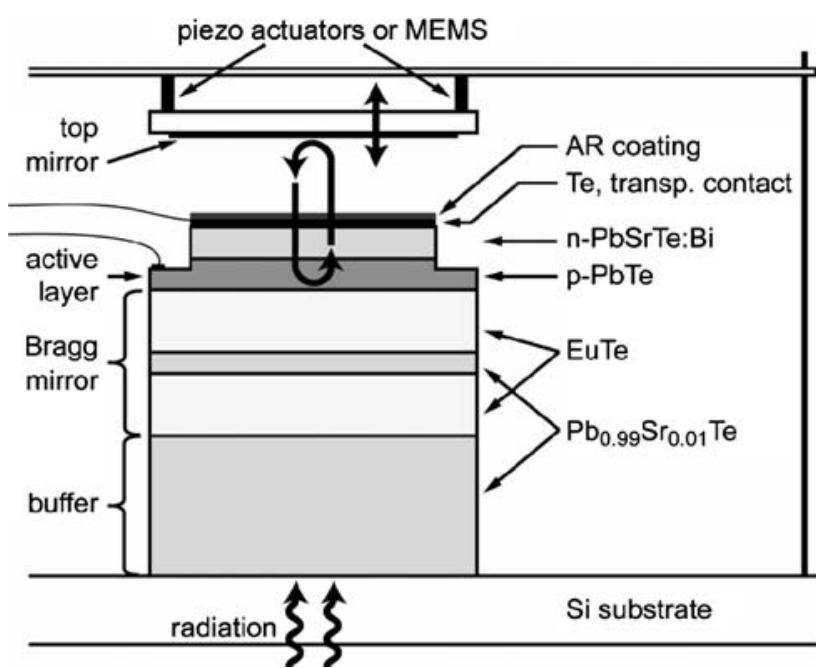

Fig. 1. Tunable resonant cavity enhanced detector (RCED) realized with IV-VI materials on a Si substrate. The top mirror is fabricated with piezo-actuators or MEMS technology and hybridized to the detector chip. ${ }^{10}$

lattice-matched II-VI broadband IR detectors and, at operation temperatures $>200 \mathrm{~K}$, even above the theoretical diffusion-noise limit for "thick" IR-detectors with the same cut-off wavelength. In addition, we realized wavelength tunable sensors by using a hybrid cavity with variable length using either piezo-actuators or micro-electromechanical systems (MEMS) technologies. ${ }^{10}$

Figure 1 shows a schematic cross section. The active layer forms a $n^{+}-p$ structure with a thin $(0.3 \mu \mathrm{m}) \mathrm{PbTe}$ active layer. It is grown on a 1.5 pair Bragg mirror. The top contact is transparent to the IR radiation to be recorded. Figure 2 shows spectra obtained with piezo-actuators at three different cavity lengths. The spectrum exhibits only one peak, which can be tuned by displacing the top mirror. The cavity is designed for a cavity mode of order $m=16$. Lower modes (at longer wavelengths) are not recorded due to the cut-off wavelength chosen, while higher-order modes are suppressed by appropriately choosing the compositions $x$ of the $\mathrm{Pb}_{1-x} \mathrm{Sr}_{x}$ Te buffer layers. This limits the range over which a single line is obtained. Figure 3 shows the total measured range over which tuning is possible for a different device.

Devices with movable MEMS mirrors were also realized. The MEMS mirror consists of a gold-coated square mirror plate attached to four symmetrically arranged suspension legs. ${ }^{11}$ It is displaced by electrostatic actuation. The counterelectrodes are placed on a glass support wafer at a distance of $10 \mu \mathrm{m}$. The working principle and images of the MEMS mirror in the realized device are shown in Fig. 4. A movement of more than $3 \mu \mathrm{m}$ is achieved by applying a voltage of $30 \mathrm{~V}$ between the silicon mirror membrane and the electrodes on the glass wafer. The MEMS mirror is then integrated with the IR diode and Bragg mirror to form a complete

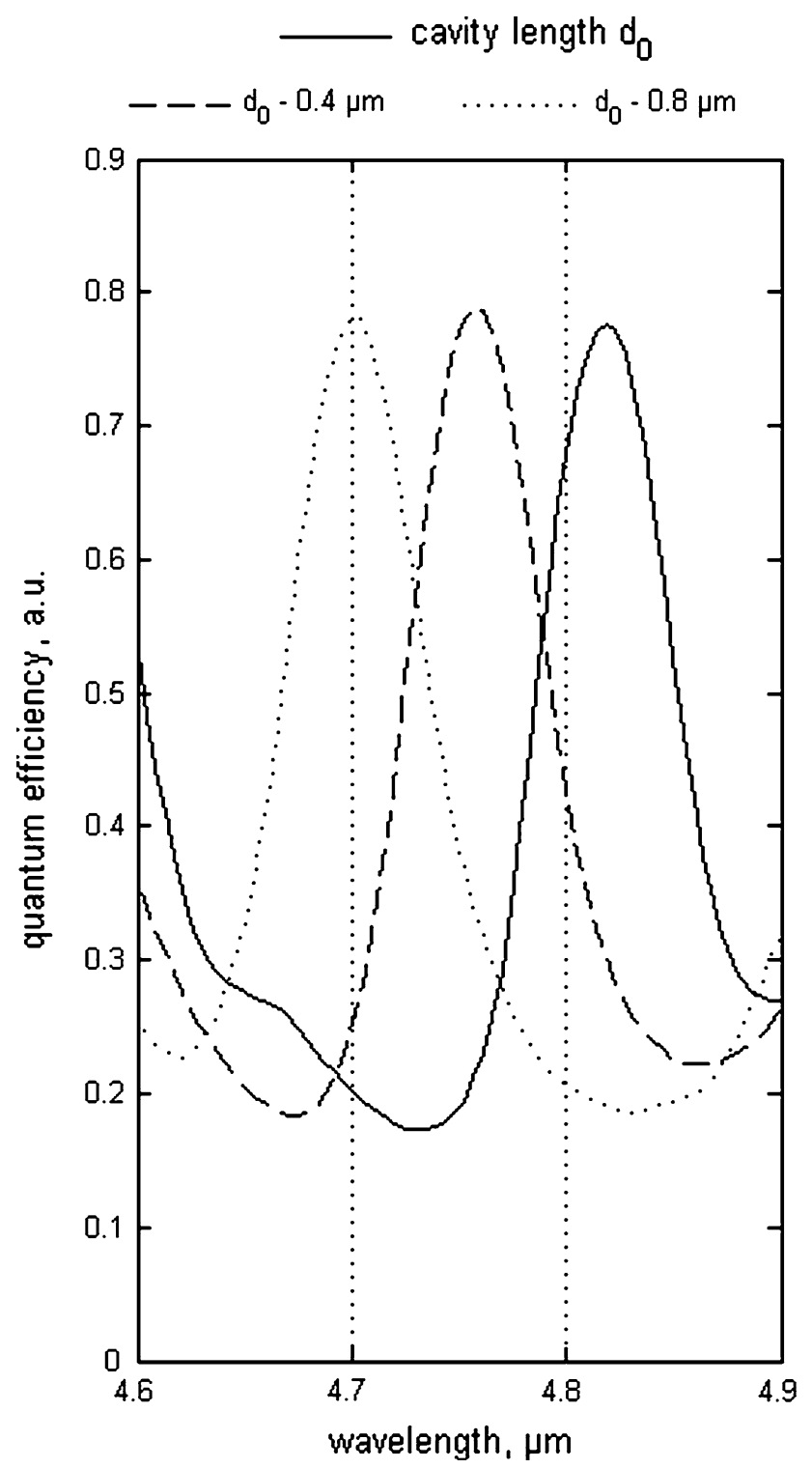

Fig. 2. Three spectra with different cavity lengths obtained with the piezo-actuated mirror; $T=100 \mathrm{~K}^{10}$

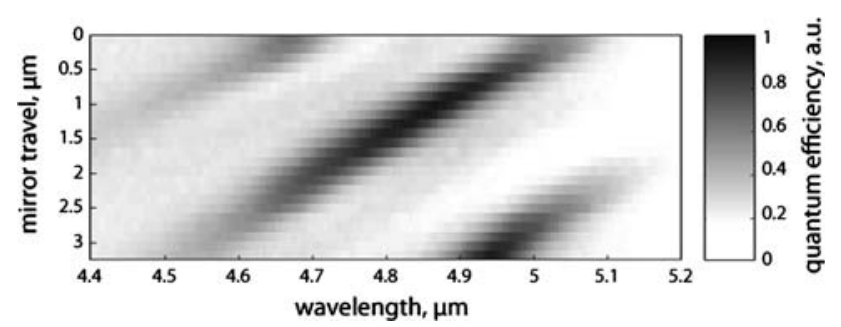

Fig. 3. Tuning range showing the different cavity modes for a different RCED.

MEMS tunable RCED. ${ }^{12}$ Figure 5 shows a spectrum obtained with the MEMS mirror. Here, three cavity modes appear instead of one only. This is due to a too high cavity length with respect to the intended design, caused by a technical error. 


\section{(a)}
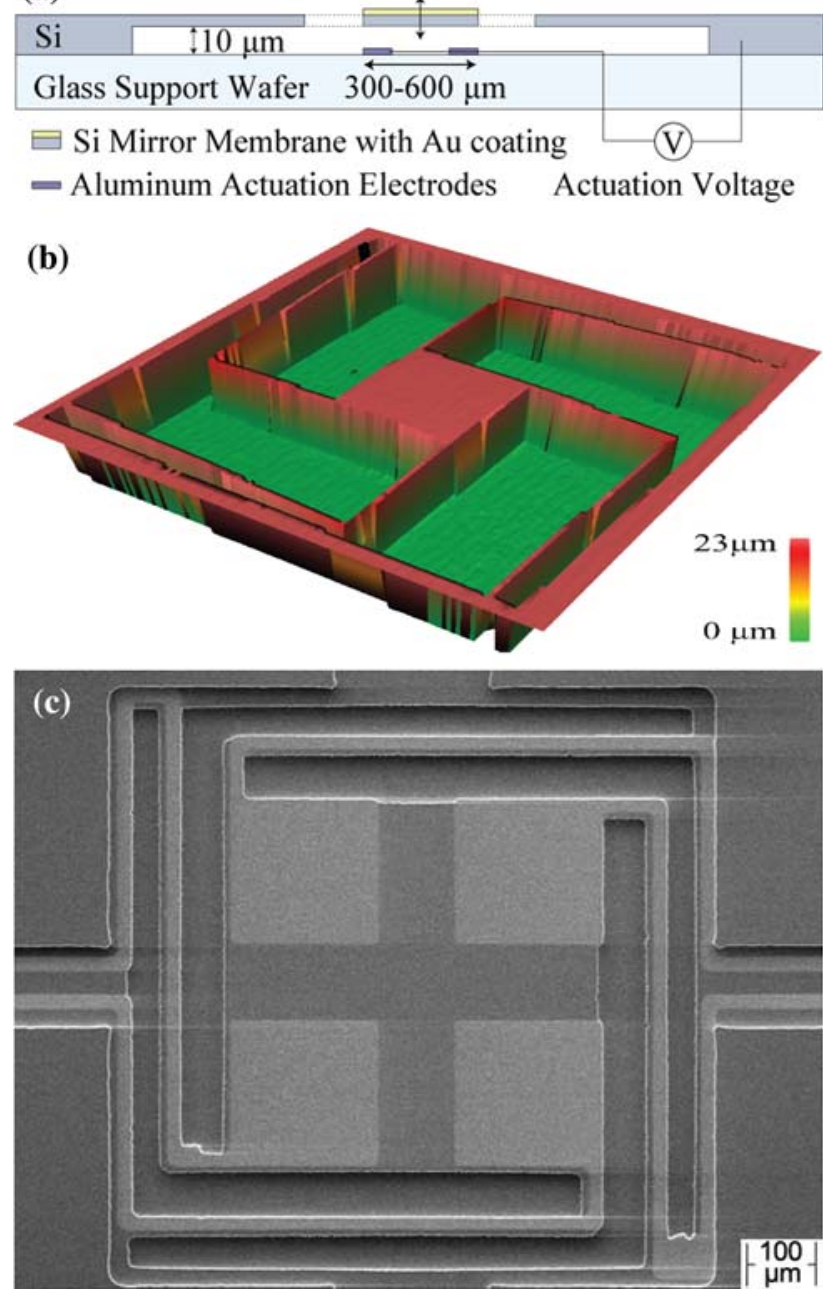

Fig. 4. MEMS mirror for the tunable IV-VI RCED on an Si substrate. (a) Working principle of the MEMS mirror. The mirror is displaced by applying a voltage between the actuation electrodes and the silicon mirror membrane. (b) White-light interferometer image of a realized freestanding micromirror, and (c) scanning electron microscopy (SEM) picture of a mirror membrane etched in the silicon substrate.

In Fig. 6, the experimental $R_{0} A$ values are plotted (points) together with the theoretical curves. For a thick abrupt $n^{+}-p$ photodiode without surface recombination, the diffusion current limit is given by Rogalski et al. as ${ }^{1}$

$$
R_{\mathrm{o}} A_{\mathrm{D}}=k T / q^{2} \cdot N_{\mathrm{a}} / n_{\mathrm{i}}^{2} \cdot \tau_{\mathrm{e}} / L
$$

where $T$ is temperature, $k$ Boltzmann's constant, $q$ electron charge, $N_{\mathrm{a}}$ the acceptor density, $n_{\mathrm{i}}$ the intrinsic density, and $\tau_{\mathrm{e}}$ the electron lifetime. For a "thick" diode, where the thickness $L$ of the absorbing layer is optimised with respect to light absorption, $L=L_{\mathrm{e}}=\left(D \tau_{\mathrm{e}}\right)^{1 / 2}$, where $L_{\mathrm{e}}$ is the electron diffusion length and $D$ the diffusion coefficient. The strong temperature dependence is mainly caused by the $n_{\mathrm{i}}^{-2}$, which is proportional to $\mathrm{e}^{E_{\mathrm{g}} / k T}$, where $E_{\mathrm{g}}$ is the bandgap energy. $\tau_{\mathrm{e}}$ is limited by Auger recombination. In this case $R_{\mathrm{o}} A_{\mathrm{D}}$ is independent of $N_{\mathrm{a}}$ for

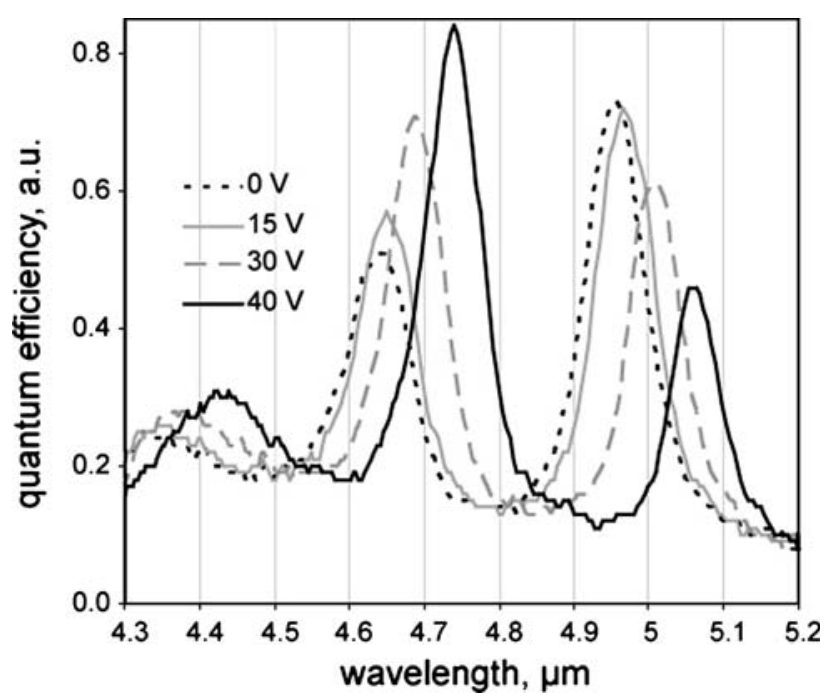

Fig. 5. Spectra with different cavity lengths obtained with the MEMS mirror; $T=100 \mathrm{~K}$. Lines of multiple order appear due to a too long cavity length with respect to the design.

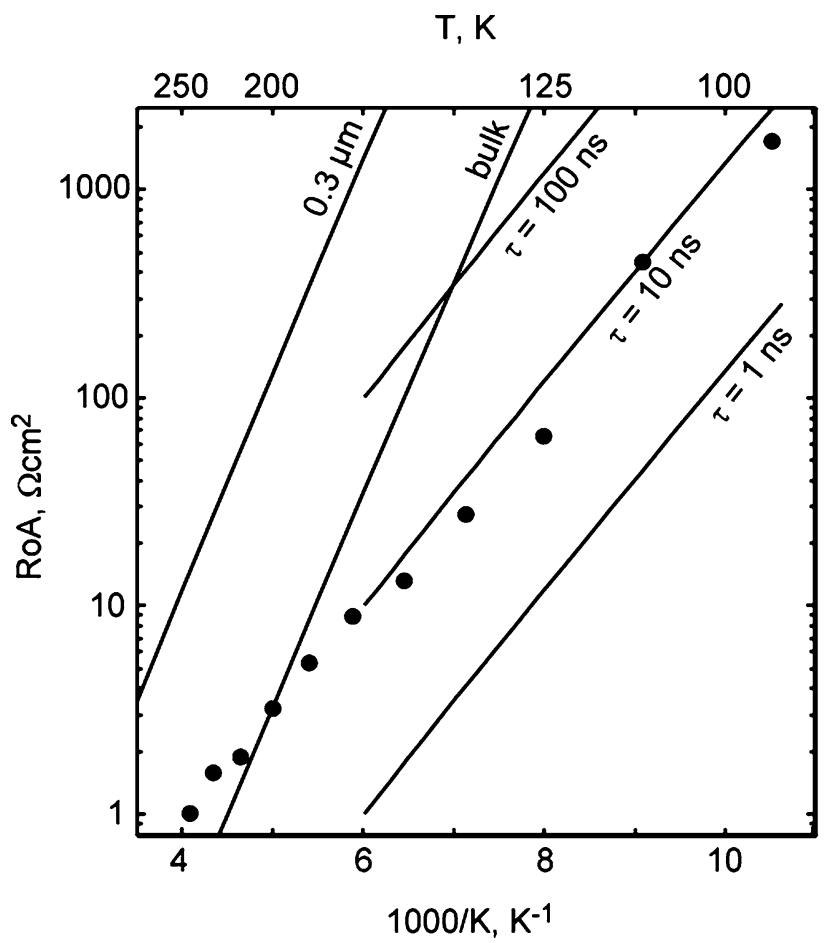

Fig. 6. Resistance-area product at zero bias of the RCED versus temperature (see text). ${ }^{10}$

the doping in the $10^{17} \mathrm{~cm}^{-3}$ range employed in our devices $\left(N_{\mathrm{a}}\right.$ in Eq. (1) is cancelled by the $N_{\mathrm{a}}$ dependence of $\tau_{\mathrm{e}}$ ). This curve is labeled "bulk" in Fig. 6 and represents the ultimate limit of a conventional infrared photodiode.

If the thickness $L$ of the absorbing layer is $L \ll L_{\mathrm{e}}$ and without surface recombination, higher $R_{\mathrm{o}} A$ values result. The calculated line in Fig. 6 for a 
0.3- $\mu \mathrm{m}$ thin $\mathrm{PbTe}$ absorber layer as used for the present device shows an approximately 40 times increased theoretical $R_{0} A$ value. Quantum efficiencies in such thin layers would decrease to unacceptable small values in conventional photodiodes. However, due to the cavity effect in RCEDs, high quantum efficiencies result since a photon which enters the cavity travels back and forth many times until it is absorbed.

The experimental points as indicated in Fig. 6 were derived from fitted current-voltage curves and corrected for series resistances. At $250 \mathrm{~K}$ to $200 \mathrm{~K}$, they are above the values for an optimized bulk photodiode, demonstrating the potential of the RCED principle. However, the values are still much below the theoretical thin limit. This is due to structural defects in the active layer, while surface recombination effects play a minor role.

The slope of the experimental $R_{0} A$ values is about half of that of the calculated diffusion limit, however the diodes are limited by Shockley-Read recombination at defects in the depletion region. The $R_{\mathrm{o}} A$ product for this case is ${ }^{1}$

$$
R_{\mathrm{o}} A_{\mathrm{SR}}=2 k T / q \cdot \ln \left(N_{\mathrm{a}} N_{\mathrm{d}} / n_{\mathrm{i}}^{2}\right) / n_{\mathrm{i}} \cdot \tau_{\mathrm{SR}} / w
$$

Here $N_{\mathrm{d}}$ is the donor density, $\tau_{\mathrm{SR}}$ the ShockleyRead lifetime, and $w$ the width of the depletion region (about $0.2 \mu \mathrm{m}$ in our case). $R_{\mathrm{o}} A_{\mathrm{SR}}$ curves calculated for three different $\tau_{\mathrm{SR}}$ are also plotted in Fig. 6. It follows that the experimental values are determined by $\tau_{\mathrm{SR}} \approx 10 \mathrm{~ns}$. These values are much higher as compared to PbTe bulk photodiodes we fabricated on Si substrates. We may conclude that this is due to the limited thickness (volume) of the active device part where SR generation/recombination noise of charge carriers is possible. With RCEDs, it is therefore possible to obtain higher sensitivities even with less perfect layers.

\section{VERTICAL EXTERNAL-CAVITY SURFACE-EMITTING LASERS}

Vertical external-cavity surface-emitting lasers (VECSEL) are currently of high interest due to their good beam quality, high power, and power scalability as well as wavelength tunability. ${ }^{13}$ They are optically pumped in most cases and have been realized employing multiple-quantum-well structures with GaAs, InP, or GaSb-based III-V compounds. The latter are suited for longer wavelengths; VECSELs emitting wavelengths up to $\sim 2.3 \mu \mathrm{m}^{14-16}$ have been realized. This appears to be the longest wavelength obtained with a VECSEL up to now (quantum cascade lasers which emit at longer wavelengths are edge emitting, and therefore have low beam quality). Monolithic IV-VI vertical-cavity surface-emitting laser (VCSELs) that are optically pumped and emit in the $4 \mu \mathrm{m}$ to $7 \mu \mathrm{m}$ range, and, in pulsed mode, up to above room temperature (RT) have been described. ${ }^{17-20}$ IV-VI materials are well suited to real- ize VCSELs as well as VECSEL structures due to the ease of fabricating Bragg mirrors.

Here, we describe the first VECSELs emitting above a wavelength of $5 \mu \mathrm{m}$. Figure 7 shows schematic cross sections of two types of devices realized. The resonant cavity is formed between the bottom Bragg mirror and the top curved mirror. As a transparent substrate, $\mathrm{BaF}_{2}(111)$ is used and placed inside the cavity [we have not used $\mathrm{Si}$ as a substrate to date because of its large refractive index, which would necessitate an additional efficient antireflection (AR) coating]. For device (a), the gain layer consists of just $2 \mu \mathrm{m}$ of $\mathrm{PbTe}$. It is grown on an $\mathrm{Pb}_{0.8} \mathrm{Eu}_{0.2}$ Te AR layer. For device (b), two $150-\mathrm{nm}$ thin $\mathrm{PbTe}$ gain layers are separated by $\mathrm{Pb}_{1-x} \mathrm{Eu}_{x} \mathrm{Te}$ barrier layers.

For both device types, the gain structures are followed by a two-pair Bragg mirror with alternating $\mathrm{Pb}_{0.93} \mathrm{Eu}_{0.07} \mathrm{Te} / \mathrm{BaF}_{2}$ quarter-wavelength layers. Its reflectance is $>99 \%$ at the design wavelength of $5.3 \mu \mathrm{m}$ (corresponding to operation at about $95 \mathrm{~K}$ for $\mathrm{PbTe}$ ).

The insets in Fig. 7 show the calculated intensity profiles at a wavelength of $5.3 \mu \mathrm{m}$. The two $\mathrm{PbTe}$ gain layers of structure (b) are located at the waists of the standing-wave pattern. Here, the composition of the first barrier is $x=0.2$, which is transparent to the pump beam. For the composition of the second barrier, $x=0.07$ was chosen. The remaining pump beam is absorbed in this layer, but this composition leads to higher refractive index and facilitates obtaining better structural quality of the next layers, thus finally resulting in better reflectivity of the Bragg mirror.

The top curved mirror, again with reflectivity $>99 \%$, consists of two pairs of $\mathrm{SiO}_{2} / \mathrm{Si}$ and a $\mathrm{Cr}$ intermediate layer. Cavity length is slightly shorter

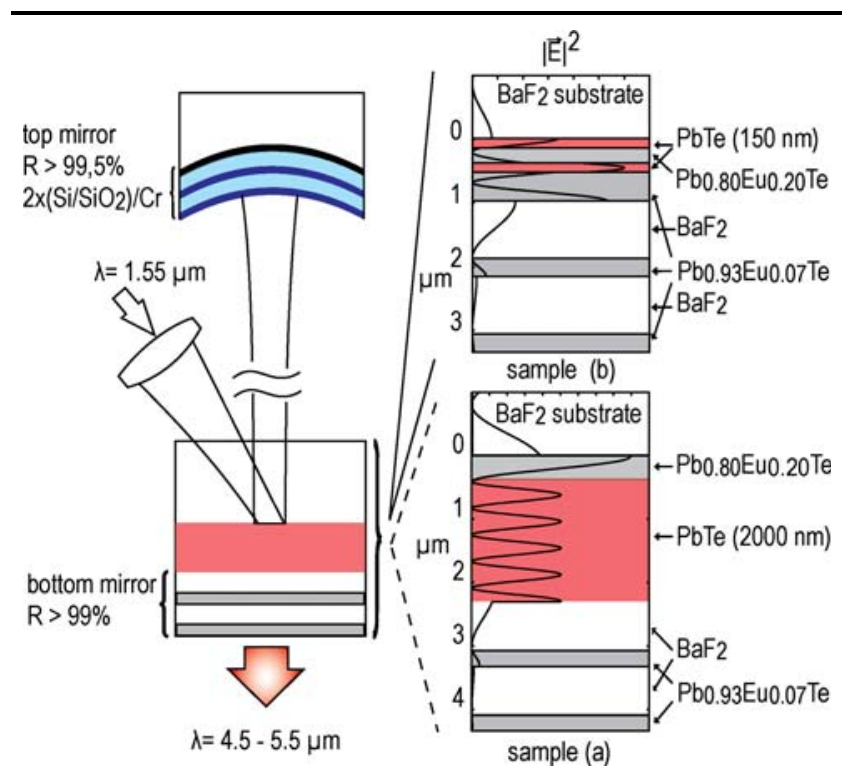

Fig. 7. Vertical external-cavity surface-emitting laser (VECSEL), schematic cross sections for two different gain structures. ${ }^{23}$ 

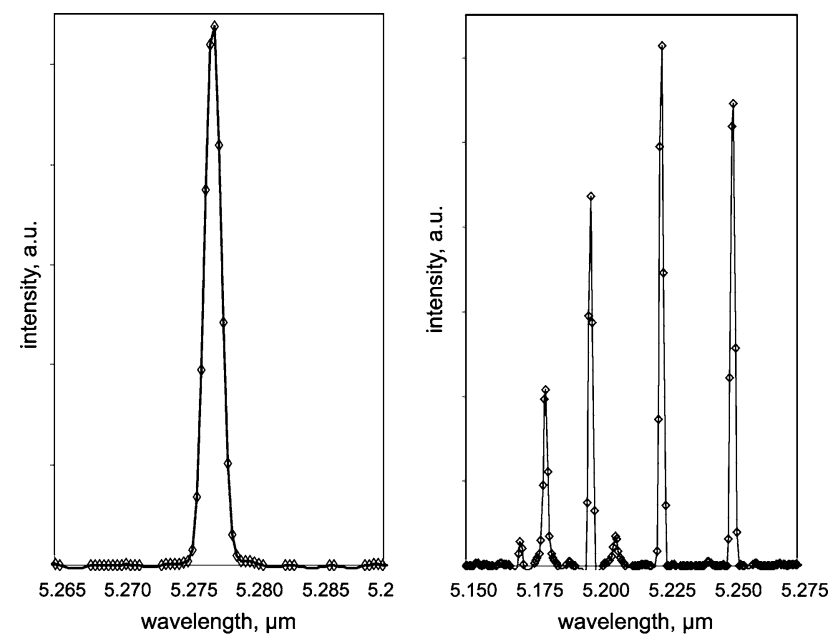

Fig. 8. Vertical external-cavity surface-emitting laser (VECSEL). Left: monomode emission spectrum at low pump power; right: multimode operation at higher pump power, $T=100 \mathrm{~K}$.

than the radius of curvature of this top mirror $(25 \mathrm{~mm})$ to obtain a stable resonator with a suitable mode diameter. The exciting $1.5-\mu \mathrm{m}$ laser beam is focused and adjusted to pump through the $\mathrm{BaF}_{2}$ substrate and to match the pumped area with the VECSEL mode size $(\sim 200 \mu \mathrm{m}$ diameter $)$.

For both structures, as shown in Fig. 8, the emission spectrum of the VECSEL operating at $100 \mathrm{~K}$ is monomode for lower excitation power, while the second spectrum taken after increasing the pumping power is multimode and complicated due to the composed resonator. The shift to lower wavelengths in the second spectrum is caused by heating. Linewidths are $<0.4 \mathrm{~cm}^{-1}$, limited by the resolution of the infrared spectrometer used. A beam quality as good as $M^{2}=1.3$ has been deduced from the measured angular spread and (calculated) diameter of the fundamental mode at the exit of the cavity.

Figure 9 (left) shows the light in/out characteristics of the two structures in pulsed mode with $3 \mu \mathrm{s}$ pulse widths. For structure (a) with $2 \mu \mathrm{m} \mathrm{PbTe}$ gain layer, output powers up to $\sim 45 \mathrm{~mW}_{\mathrm{p}}$ are observed. No thermal rollover occurs up to the highest excitation (limited by the pump laser). In contrast, structure (b) with the two thin gain layers shows higher output power at lower excitation and higher differential efficiency. However, thermal rollover limits the output power to somewhat above $50 \mathrm{~mW}_{\mathrm{p}}$ at these rather long pulse widths.

Continuous wave (CW) emission has been obtained with the two-gain-layer structure (b). Maximum emission power was $1 \mathrm{~mW}$ to $3 \mathrm{~mW}$ at $95 \mathrm{~K}$ operation temperature, sensitively depending on precise alignment of the cavity mirrors and pump beam. The right of Fig. 9 shows light in/out characteristics for quasi-CW excitation with $800 \mu \mathrm{s}$ pulse widths and 50\% duty cycle. Threshold pump power is as low as $\sim 300 \mathrm{~mW}\left(800 \mathrm{~W}_{\mathrm{p}} / \mathrm{cm}^{2}\right)$, while

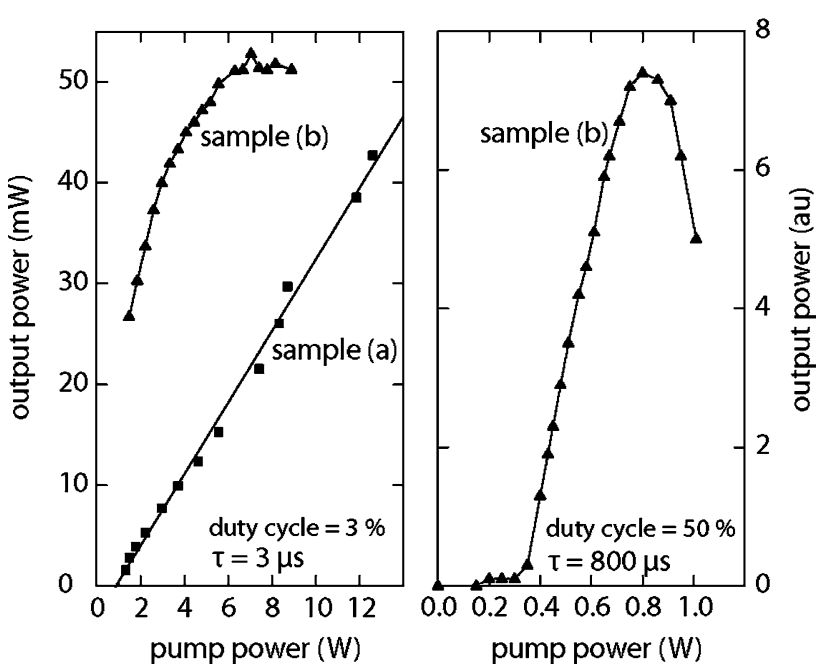

Fig. 9. Light in/out characteristics at $100 \mathrm{~K}$ for the two structures and different duty cycles. ${ }^{23}$

thermal rollover occurs already at $800 \mathrm{~mW}$ pump power. Differential quantum efficiency is $\sim 2 \%$.

The difference in the lasing behaviour between structures (a) and (b) may be explained by recalling the strong temperature dependence of the emission wavelengths of $\mathrm{PbTe}(\sim 10 \mathrm{~nm} / \mathrm{K})$ : For structure (b), a temperature change away from the design wavelength shifts the position of the beam waists out of the thin PbTe gain layers. This results in decreased gain already at lower pump powers compared to structure (a) due to heating effects. On the contrary, the gain in structure (b) is more efficient than in structure (a), leading to a higher differential quantum efficiency. Structure (a) with just a thick gain layer is less sensitive to changes in beam waist positions due to changed emission wavelengths, therefore lasing is observed over a larger temperature range up to $\sim 140 \mathrm{~K}$. In contrast, structure (b) lases up to $\sim 120 \mathrm{~K}$ only (not shown).

At the present stage of development, the laser may be applied for high-sensitivity spectroscopy where the detector has to be liquid-nitrogen cooled. Detector and laser may therefore be placed in the same dewar. For operation at higher temperature, due to the considerable temperature dependence of the bandgap of IV-VI materials, the design for the resonant periodic gain part in structure (b), Bragg mirror and AR coating has to be adapted to fit the emission wavelength at that temperature. In addition, we expect significantly increased operation temperatures and emission powers if efficient heat removal precautions are taken. These may include direct growth on Si substrates (as used for edgeemitting IV-VI lasers ${ }^{21}$ ) or epitaxial lift-off and liquid capillary bonding to $\mathrm{SiC}$ or diamond substrates.

According to the low Auger recombination of narrow-gap IV-VI materials, even CW operation up to room temperature is feasible at least for not too 
high emission wavelengths. ${ }^{22}$ Experimentally, above-RT operation was demonstrated in pulsed mode with pulses in the ns range. Quantum well (QW) structures should allow lower thresholds. However, a comparison of different IV-VI VCSELs did not show a significant difference between bulk, QW or quantum-dot IV-VI lasers. ${ }^{18}$ The performance of all IV-VI lasers described to date seems to be limited by defects (dominated by Shockley-Read recombination). Compared to theory, the threshold power observed for optically pumped edge-emitting IV-VI lasers was estimated to be about 100 times higher then the fundamental Auger limit. ${ }^{21}$ The present VECSEL shows about ten times lower threshold power compared to that work, at least partly due to improved material quality. ${ }^{23}$ However, further optimization will further decrease threshold powers and therefore increase operating temperature.

\section{CONCLUSIONS}

Narrow-bandgap IV-VI (lead chalcogenide) semiconductors are suited to be grown lattice-mismatched on $\mathrm{Si}(111)$ and other substrates and to fabricate infrared optoelectronic devices covering the wavelength range from $<3 \mu \mathrm{m}$ to $>30 \mu \mathrm{m}$. In addition, epitaxial Bragg mirrors can easily be grown with these materials. Even with only a few pairs of quarter-wavelength high/low index layers, the mirrors exhibit high reflectivity and a broad stop band. By combining these facts, two new type of devices have been realized for the first time in the mid-IR range using $\mathrm{PbTe}$ as active narrow-bandgap material.

Tunable RCEDs exhibit narrow line widths, tuning range is in the $4 \mu \mathrm{m}$ to $5.5 \mu \mathrm{m}$ wavelength range, and they show high quantum efficiencies and their noise currents are below the limit of bulk devices at operating temperatures above $200 \mathrm{~K}$.

Vertical external-cavity surface-emitting lasers pumped optically at $1.5 \mu \mathrm{m}$ wavelength emit above $5 \mu \mathrm{m}$. The spectrum is monomode at lower excitation power, while it is multimode at higher powers. At $\sim 100 \mathrm{~K}$ operating temperature, output power is up to $50 \mathrm{~mW}$ with pulsed excitation, and up to $3 \mathrm{~mW}$ in $\mathrm{CW}$ operation. Threshold power is as low as $\sim 300 \mathrm{~mW}$. No precautions for efficient heat removal have been taken to date; a significant improvement of the parameters is expected if heat spreaders are employed.

\section{REFERENCES}

1. A. Rogalski, K. Adamiec, and J. Rutkowski, Narrow Gap Semiconductor Photodiodes (Bellingham, WA: SPIE, 2000).

2. J. Faist, F. Capasso, D.L. Sivco, C. Sirtori, A.L. Hutchinson, and A.Y. Cho, Science 264, 553 (1994).

3. P. Müller, H. Zogg, A. Fach, J. John, C. Paglino, A.N. Tiwari, M. Krejci, and G. Kostorz, Phys. Rev. Lett. 78, 3007 (1997).

4. H. Zogg, S. Blunier, T. Hoshino, C. Maissen, J. Masek, and A.N. Tiwari, IEEE Trans. Electron. Dev. 38, 1110 (1991).

5. H. Zogg, K. Alchalabi, D. Zimin, and K. Kellermann, IEEE Trans. Electron. Dev. 50, 209 (2003).

6. W. Heiss, T. Schwarzl, and G. Springholz, Proc. 9th Int. Conf. on Narrow Gap Semiconductors, Berlin, ed. N. Puhlmann, H.-U. Müller, and M. von Ortenberg, 1999, p. 61.

7. M.S. Unlü and S. Strite, J. Appl. Phys. 78, 607 (1995).

8. C.A. Musca, J. Antoszewski, K.J. Winchester, A.J. Keating, T. Nguyen, K.K.M.B.D. Silva, J.M. Dell, L. Faraone, P. Mitra, J.D. Beck, M.R. Skokan, and J.E. Robinson, IEEE Electron. Dev. Lett. 26, 888 (2005).

9. M. Arnold, D. Zimin, and H. Zogg, Appl. Phys. Lett. 87, 141103 (2005)

10. F. Felder, M. Arnold, M. Rahim, C. Ebneter, and H. Zogg, Appl. Phys. Lett. 91, 101102 (2007).

11. N. Quack, S. Blunier, J. Dual, M. Arnold, F. Felder, C. Ebneter, M. Rahim, and H. Zogg, Sensor Actuat. A Phys. 143, 29-33 (2008)

12. N. Quack, S. Blunier, J. Dual, M. Arnold, F. Felder, C. Ebneter, M. Rahim, and H. Zogg, J. Opt. A: Pure Appl. Opt. 10, 044015 (2008).

13. M. Kuznetsov, F. Hakimi, R. Sprague, and A. Mooradian, IEEE Photon. Technol. Lett. 9, 1063 (1997).

14. J.M. Hopkins, A.J. Maclean, E. Riis, D. Burns, N. Schulz, M. Rattunde, C. Manz, K. Köhler, and J. Wagner, Opt. Exp. 15, 8212 (2007).

15. J. Wagner, N. Schulz, M. Rattunde, C. Ritzenthaler, C. Manz, C. Wild, and K. Köhler, Phys. Stat. Sol. (c) 4, 1597 (2007).

16. A. Ouvrard, A. Garnache, L. Cerutti, F. Genty, and D. Romanini, IEEE Photon. Technol. Lett. 17, 2020 (2005).

17. J. Fürst, H. Pascher, T. Schwarzl, M. Böberl, W. Heiss, G. Springholz, and G. Bauer, Appl. Phys. Lett. 81, 208 (2002).

18. T. Schwarzl, G. Springholz, M. Böberl, E. Kaufmann, J. Roither, W. Heiss, J. Fürst, and H. Pascher, Appl. Phys. Lett. 86, 031102 (2005).

19. H.Z. Xu, F. Zhao, A. Majumdar, and Z. Shi, Electron. Lett. 39,659 (2003)

20. F. Zhao, H. Wu, L. Jayasinghe, and Z. Shi, Appl. Phys. Lett. 80, 1129 (2002).

21. K. Kellermann, D. Zimin, K. Alchalabi, P. Gasser, N.A. Pikhtin, and H. Zogg, J. Appl. Phys. 94, 7053 (2003).

22. S. Khosravani and Z. Shi, Appl. Phys. Lett. 78, 139 (2001).

23. M. Rahim, M. Arnold, F. Felder, K. Behfar, and H. Zogg, Appl. Phys. Lett. 91, 151102 (2007). 